\title{
Evaluation of portion size estimation aids among Irish consumers
}

\author{
L. K. Pourshahidi ${ }^{1}$, G. P. Faulkner ${ }^{1}$, M. Spence ${ }^{2}$, M. Dean ${ }^{2}$, S. O’Brien ${ }^{3}$, M. Goulding ${ }^{3}$, E. R. Gibney ${ }^{3}$, \\ M. A. Kerr ${ }^{1}$, T. A. McCaffrey ${ }^{1}$, J. M. W. Wallace ${ }^{1}$ and M. B. E. Livingstone ${ }^{1}$ \\ ${ }^{1}$ Northern Ireland Centre for Food and Health, University of Ulster, Coleraine, BT52 1SA, ${ }^{2}$ School of Biological Sciences, \\ Queen's University Belfast, BT9 5AG and ${ }^{3}$ Institute of Food and Health, University College Dublin, Belfield, \\ Dublin 4, Ireland
}

Larger food portion sizes (PS) have been identified as one of the many environmental factors associated with obesity ${ }^{(1)}$. The increasing PS of foods available in the marketplace have influenced the amount of food being served in the home ${ }^{(2,3)}$, highlighting the need for guidance to help consumers effectively manage their food PS in this context. Therefore, the aim of the current study was to evaluate the perceived acceptability and efficacy of a range of portion size estimation aids (PSEA) among Irish consumers.

Sixteen commonly consumed foods were selected, to include a diversity of shapes (e.g. slices, chunks, whole) and visual characteristics (e.g. solids, amorphous, liquids). The PSEA evaluated were; measuring scales/jug, reference objects, household measures and pack markings/labels. Adults (18-64 years), living on the island of Ireland were recruited to take part in the study. For each food, participants were shown the PSEA, before being asked to serve out the recommended PS of that food, using the aid they considered most appropriate. Participants were asked to rate their preferred aid in terms of its ease of use ( $1=$ not very easy to $5=$ very easy) and likelihood of future use $(1=$ not likely to 5 = very likely). Participants were asked to choose from a list of situations/eating occasions where they would be most likely to use the PSEA. Lastly, any problems encountered whilst using the PSEA were also noted as an open response.

A total of 120 adults (61 males; 59 females) completed the study. The group were mainly aged 18-25 years (50.0\%); normal weight (self-reported BMI $\left.18.5-24.9 \mathrm{~kg} / \mathrm{m}^{2}\right)(63.2 \%)$; postgraduate/undergraduate students $(50.8 \%)$; following no special diet $(88.3 \%)$.

\begin{tabular}{|c|c|c|c|c|c|c|c|}
\hline \multirow[b]{2}{*}{ PSEA } & \multirow{2}{*}{$\frac{\text { Preference* }}{\%}$} & \multicolumn{3}{|c|}{ Ease of use $\dagger$} & \multicolumn{3}{|c|}{ Likelihood of future use $\dagger$} \\
\hline & & $1-2$ & 3 & $4-5$ & $1-2$ & 3 & $4-5$ \\
\hline Measuring scales/jug & 17 & 8 & 13 & 79 & 43 & 17 & 40 \\
\hline Reference objects & 44 & 4 & 18 & 78 & 28 & 18 & 54 \\
\hline Household measures/utensils & 50 & 3 & 7 & 90 & 18 & 13 & 69 \\
\hline Food packaging & 47 & 8 & 15 & 77 & 15 & 11 & 74 \\
\hline
\end{tabular}

*Calculated as the number of times a PSEA was selected for use, divided by the number of times it was available as an option, and multiplied by 100 . $†$ Values shown are $\%$ within row for each rating.

Overall, the measuring scales/jug were the least preferred aid compared to the other PSEA. Despite the majority of participants rating the measuring scales/jug as either easy/very easy to use (rating 4-5 out of 5), a large proportion were not likely to use them again (rating 1-2 out of 5). The other PSEA were rated highly by the majority in terms of ease of use and likelihood of future use. Participants unanimously agreed that they would be most likely to use PSEA when preparing food in the home, particularly for dinner, compared to any other eating occasion. Perceptions that the PSEA are inconvenient to use overall, or not practical for certain foods, were commonly cited barriers to their use. In this study while PSEA were rated highly for ease of use, this did not always translate into likelihood of future use. Barriers to their uptake should be addressed in future consumer guidance on effective management of PS selection.

This is based upon works supported by safefood, the Food Safety Promotion Board, under Grant No. 07-2010. Ethical approval was obtained from the Biomedical Sciences Ethics Filter Committee, University of Ulster and the study was conducted according to the guidelines laid down in the Declaration of Helsinki. We acknowledge Miss Ciara McGoldrick for her help with data collection.

1. McCarthy SN, Robson PJ, Livingstone MBE, et al. (2006) Int J Obes 30, 993-1002.

2. Young LR and Nestle M (2003) J Am Diet Assoc 103, 231-4.

3. Wrieden W, Gregor A and Barton K (2008) Proc Nut Soc 67, E211. 